March 1997; Revised March 1998

UM-P-97/09

RCHEP $97 / 02$

\title{
Maximal mixing neutrino models
}

\author{
J. P. Bowes and R. R. Volkas \\ School of Physics \\ Research Centre for High Energy Physics \\ The University of Melbourne \\ Parkville 3052 Australia.
}

\begin{abstract}
We account for the solar and atmospheric neutrino problems by introducing maximal mixing between conventional neutrinos and sterile neutrino partners. We achieve this by invoking a seesaw-like mechanism which not only provides us with maximally mixed neutrino/sterile-neutrino mass eigenstates but also accounts for the relative suppression of the neutrino masses compared to the charged fermion masses. In obtaining such an extended seesaw mechanism we are required to introduce a new $U(1)$ global symmetry together with an extended Higgs sector.
\end{abstract}

\section{Introduction and Motivation}

The physics of neutrinos continues to be very exciting. Four experiments (GALLEX, SAGE, Kamiokande and Homestake) have observed a deficit in the solar neutrino flux 1 . Preliminary results from SuperKamiokande are consistent with the Kamiokande measurements, further strengthening the case for the existence of a solar neutrino problem2. The most likely expla- 
nation of these results lies in electron-neutrino oscillations into other species. Substantial evidence also exists for an atmospheric neutrino problem 3 . In particular Kamiokande and Soudan II see clear evidence of an anomalously low ratio of muon-like events to electronlike events. SuperKamiokande data have confirmed this anomaly, and provided significantly stronger evidence for a zenith-angle dependence in the atmospheric muon-neutrino flux consistent with an oscillation based explanation日. The LSND Collaboration has reported evidence for both $\bar{\nu}_{\mu}-\bar{\nu}_{e}$ and $\nu_{\mu}-\nu_{e}$ oscillations 5 . This result needs to be checked by an independent experiment, such as the upgraded KARMEN 6 .

The purpose of the present paper is to construct sensible models which explain all of the neutrino results using three active and three sterile neutrino flavours. In order to motivate the introduction of sterile neutrinos, we first briefly review the valiant attempts that have been made to explain all of the results using oscillations amongst the three known neutrino flavours: $\nu_{e}, \nu_{\mu}$ and $\nu_{\tau}$. The Cardall and Fuller schemed sees the atmospheric and LSND results accounted for through a single $\delta \mathrm{m}^{2}$ of about $0.3 \mathrm{eV}^{2}$, with the solar results related to another $\delta m^{2}$ of about $10^{-5} \mathrm{eV}^{2}$. The atmospheric neutrino anomaly is handled through large-angle $\nu_{\mu}-\nu_{\tau}$ oscillations. However, the large mass splitting required renders the atmospheric $\nu_{\mu}$ flux zenith-angle independent. This is disfavoured by the preliminary SuperKamiokande data. The Acker and Pakvasa three-flavour scheme 8 , by constrast, accounts for the atmospheric anomaly through large angle $\nu_{\mu}-\nu_{e}$ oscillations. This scenario is disfavoured by the recent CHOOZ reactor-based bounds 9 (the Palo Verde reactor experiment will check this result 10 ). It is also disfavoured by the zenith-angle flux dependences revealed by the preliminary SuperKamiokande datat 11.

Another interesting three-flavour scenario is the maximal mixing model of Harrison, Perkins and Scott12. This theoretically appealing scheme solves the solar neutrino problem through an energy-independent flux reduction by a factor of 5/9. The atmospheric neutrino problem is also solved provided an appropriate $\delta m^{2}$ is used (for an update see Ref.11). However, this model has difficulty accounting for the LSND measurements.

We will assume for the purposes of this paper that all three neutrino anomalies (solar, 
atmospheric and LSND) are real. Given this assumption, the discussion of the previous two paragraphs leads to the conclusion that the three known neutrino flavours are not sufficient to explain the totality of neutrino data. This in turn motivates the introduction of sterile neutrinos.

Degree of freedom minimality would be achieved by the introduction of only one sterile neutrino flavour $\nu_{s}$. Two different scenarios result: (i) 13 the solar neutrino problem can be solved through MSW 1 enhanced small angle $\nu_{e}-\nu_{s}$ oscillations with $\delta m_{e s}^{2} \simeq 10^{-5} e V^{2}$. The atmospheric problem is solved by large-angle or maximal $\nu_{\mu}-\nu_{\tau}$ oscillations with $\delta m_{\mu \tau}^{2} \simeq 10^{-3}-10^{-2} e V^{2}$. The LSND result can be incorporated by small angle $\nu_{e}-\nu_{\mu}$ oscillations with $\delta m_{e \mu}^{2} \simeq 1 e V^{2}$. (ii) Alternatively, the atmospheric problem could be solved by large angle or maximal $\nu_{\mu}-\nu_{s}$ oscillations with $\delta m_{\mu s}^{2} \simeq 10^{-3}-10^{-2} e V^{2}$, while the solar problem and LSND are handled through three-flavour $\nu_{e}-\nu_{\mu}-\nu_{\tau}$ mixing with an appropriate mass and mixing angle pattern 1 .

While the introduction of only one sterile flavour is minimal in regard to degrees of freedom, it probably is not the most theoretically elegant possibility. One would have to explain why there is a mismatch between active and sterile flavours. In addition, for scenario (i) above one would need to explain why $\nu_{e}$ and $\nu_{\mu}$ have a small mixing angle whereas $\nu_{\mu}$ and $\nu_{\tau}$ have a large or maximal mixing angle. For scenario (ii) one would want to explain why the sterile flavour mixes primarily with $\nu_{\mu}$ rather than with $\nu_{e}$ or $\nu_{\tau}$, or in a comparable way with all three active flavours.

These issues of theoretical elegance motivate the introduction of three sterile flavours. We will in addition suppose that the three active flavours are mixed amongst themselves through small angles. This is motivated by both the observed pattern of small mixing in the analogous quark sector and the LSND result. The atmospheric neutrino anomaly then requires that one of the sterile flavours has a large or maximal mixing angle with $\nu_{\mu}$. A theoretical desire to maintain similarity between the generations then motivates that each of the active flavours is paired with a sterile flavour through large angle or maximal mixing. 
The neutrino sector of the Exact Parity Model contains parity or mirror partners, $\nu_{e}^{\prime}, \nu_{\mu}^{\prime}$ and $\nu_{\tau}^{\prime}$, for each of the ordinary neutrinos 16 . Since parity eigenstates must also be Hamiltonian eigenstates, maximal mixing between $\nu_{\alpha}$ and its mirror partner $\nu_{\alpha}^{\prime}(\alpha=e, \mu, \tau)$ is induced in the limit of small inter-generational mixing. The Exact Parity Model is therefore a concrete realisation of the general scenario described in the previous paragraph. The solar neutrino problem can be solved in a number of ways. (a) The first, simplest and in many ways most attractive way is via an energy independent $50 \%$ flux reduction through maximal $\nu_{e}-\nu_{e}^{\prime}$ oscillations in the large parameter range,

$10^{-10} \lesssim \delta m_{e e^{\prime}}^{2} / e V^{2} \lesssim 0.9 \times 10^{-3}$

where the upper limit is the CHOOZ $\nu_{e}$ disappearance bound. (b) Alternatively, vacuum "just-so" oscillations, which require essentially maximal mixing, can provide an energydependent flux reduction that fits the solar neutrino data well if $\delta m_{e e^{\prime}}^{2}$ is fine-tuned to the $10^{-11}-10^{-10} \mathrm{eV}^{2}$ regime 17 . (However, we disfavour this possibility because of the fine-tuning problem.) (c) It is also possible to take the inter-generational mixing parameters into the MSW range to achieve a good fit to the solar neutrino data 18 .

The atmospheric neutrino problem is analogously solved through maximal $\nu_{\mu}-\nu_{\mu}^{\prime}$ oscillations in the parameter range

$10^{-3} \lesssim \delta m_{\mu \mu^{\prime}}^{2} / e V^{2} \lesssim 10^{-2}$

(See Refs. 19 for more precise discussions of the allowed range.)

For solar neutrino scenarios (a) and (b) above, the LSND result is simply incorporated by switching on small angle mixing between $\nu_{e}$ and $\nu_{\mu}$ with $\delta m_{e \mu}^{2} \simeq 0.1-10 e V^{2}$. It is at present not clear how consistent scenario (c) above is with the LSND data.

The purpose of the present paper is to construct analogous models using strictly sterile rather than mirror neutrinos. In addition to mirror neutrinos, the Exact Parity Model features parity partners for every standard fermion, gauge boson and Higgs boson. The mirror sector particles interact amongst themselves through mirror images of the usual strong and 
electroweak interactions. The ordinary and mirror sectors interact through neutrino mixing (if neutrinos have mass), photon-mirror-photon, $Z$-mirror- $Z$ and Higgs-boson-mirror-Higgsboson mixing as well as gravitationally. The purpose of this paper is to construct a theory with essentially the same neutrino sector as the Exact Parity Model but without any of the other pieces of new physics. This is interesting to do for two main reasons. First, the discussion above has demonstrated how one arrives at essentially the Exact Parity Model neutrino sector through a combination of phenomenological and theoretical motivations without ever invoking parity symmetry as an explicit motivation. Second, it is interesting to see how elegant or inelegant a model will be which produces such a neutrino sector in the absence of exact parity symmetry. If, in a few years time, experiments unequivocally establish the existence of large angle mixing between active and sterile flavours, then the Exact Parity Model will be in competition with the models to be constructed herein. In Sec.2 we will review why maximally mixed active and sterile neutrino pairs are a natural possibility in the Standard Model with right-handed neutrinos.

Before constructing the models, two remaining issues must be briefly addressed. First, solving the solar neutrino problem via an energy-independent $50 \%$ flux reduction is considered heterodox. The main reason for this is that a comparison of Homestake with the Gallium and (Super-)Kamiokande results suggests that the mid-energy solar neutrinos, particularly the Beryllium neutrinos, should be suppressed by more than the high energy Boron and low-energy $p p$ neutrino\$20. A debate has ensued in the literature about whether or not an energy-independent suppression provides a reasonable fit to the data. Note that in addition to the present models, and the Exact Parity Model, the now disfavoured Acker-Pakvasa scheme and the Harrison, Perkins and Scott model also feature an energy independent suppression. A proper treatment of this important issue would require a lengthy discussion. For the purposes of this paper, we refer the reader instead to the extant literature22. The most important points are the following: (i) Experiments themselves will clarify the magnitude of the Beryllium neutrino flux. At present, the greater suppression claimed for Beryllium neutrinos depends crucially on the Homestake results. To be sure that the extra suppression of 
mid-energy neutrinos is real, independent experiments must confirm the Homestake result. Fortunately, the forthcoming Iodine experiment 23 and Borexino 24 will do this. (ii) Standard solar modellers do not agree on the Boron neutrino flux value, largely due to differing treatments of the $p+{ }^{7} B e \rightarrow^{8} B+\gamma$ cross-section21. It would help greatly if this theoretical uncertainty could be reduced. In the interim, our view is that any model which reduces the solar neutrino flux by a factor like $1 / 2$ or $5 / 9$, relative to the no-oscillation expectation, is worth serious consideration. (iii) If the present experimental indications in favour of an energy-dependent flux reduction are confirmed, then the "just-so" possibility discussed earlier might remain viable. Also, the introduction of inter-generational MSW transitions in addition to averaged maximal $\nu_{e}-\nu_{s}$ oscillations would remain an interesting alternative 18 .

Finally, the potential Big Bang Nucleosynthesis (BBN) problem should be addressed. Suppose both the solar and atmospheric neutrino problems are solved by maximally mixed active and sterile neutrino pairs. Then the sterile state mixing with $\nu_{\mu}$ can be brought into thermal equilibrium prior to the BBN epoch since the associated $\delta m^{2}$ is easily large enough 25. The sterile state mixing with $\nu_{e}$ destroys successful nucleosynthesis if the relevant $\delta m^{2}$ is larger than about $10^{-8} \mathrm{eV}^{2}$. Current BBN data can just tolerate four equilibrated relativistic species during the $\mathrm{BBN}$ epoch 26 . So, if the electronic $\delta m^{2}$ is less than $10^{-8}$ $\mathrm{eV}^{2}$ there is at present no problem. Otherwise, there is potentially a problem because the effective number of neutrinos would be greater than four. However, recent work has demonstrated that in a certain region of parameter space active-sterile neutrino oscillations in the early universe inevitably produce substantial neutrino chemical potentials or asymmetries 27 . This in turn suppresses active-sterile transitions via the matter-induced effective potential. Reference 28 shows that a substantial region of parameter space exists where neutrino asymmetry creation inevitably suppresses the production of the sterile states that maximally mix with $\nu_{e}$ and $\nu_{\mu}$ with $\delta m^{2}$ values motivated by the two neutrino problems. This scenario is thus cosmologically consistent due to a rather subtle but appealing piece of physics.

The remainder of this paper is structured as follows. In Sec.2 we review why maximally mixed active-sterile neutrino pairs are a natural possibility within the Standard Model aug- 
mented by right-handed neutrinos. In Sec.3 we develop mass matrices that produce both maximally mixed and light neutrinos. In Sec.4 we construct in detail a model which yields one of the suitable mass matrices, and we comment also on other possibilities. We conclude in Sec.5.

\section{Maximally mixed active and sterile neutrino pairs in the Standard Model with} right-handed neutrinos

It is interesting to note that maximal mixing between active and sterile flavours is actually a natural possibility within the Standard Model augmented by gauge-singlet right-handed neutrinos29. Consider the usual see-saw mass matrix 30 ,

$M_{\nu}=\left(\begin{array}{cc}0 & m \\ m & M\end{array}\right)$,

where $m$ is the neutrino Dirac mass and $M$ is the bare Majorana mass for the right-handed neutrino. In the see-saw model the limit $m \ll M$ is considered for exemplary reasons. However, the opposite limit $m \gg M$ is also interesting 29 . In this limit the mass eigenvalues are

$m_{1,2} \simeq m+\frac{M}{2}, \quad m-\frac{M}{2}$

and the exact mixing angle is given by

$$
\tan 2 \theta=\frac{2 m}{M}
$$

The $m \gg M$ limit therefore produces mass eigenstates that are approximately maximal mixtures of the active $\nu_{L}$ and the sterile $\left(\nu_{R}\right)^{c}$. Furthermore, the near degeneracy of the eigenvalues is correlated with near maximality for the mixing angle $(\theta \simeq \pi / 4)$ in this limit, which is an attractive feature given that both the solar and atmospheric neutrino problems suggest oscillations between nearly degenerate neutrinos.

So, one could claim that the goal of this paper is simply achieved in the above manner. However, the simple scenario above is not completely satisfactory as a theory of neutrino 
mass. As well as the hierarchy $m \gg M$ we also need $m$ to be tiny compared to the other Dirac masses in the Standard Model in order to reproduce the correspondingly tiny neutrino masses required. A satisfactory theory of neutrino mass should explain why neutrinos are much lighter than all other fermions. The standard see-saw mechanism can obviously not be used. The models constructed in this paper will incorporate an extended see-saw mechanism for producing maximally mixed active and sterile neutrinos that are both also naturally very light.

\section{Mass Matrices}

The first task in finding maximally mixed neutrino/sterile-neutrino models is to search for suitable mass matrices. As in the usual see-saw mechanism 30 these matrices will include both Dirac and Majorana mass terms, and upon diagonalisation will lead to Majorana mass eigenstates. To find acceptable maximally mixed neutrino/sterile-neutrino models we must therefore embark upon a more or less mathematical exercise in that we wish to find matrices which upon diagonalisation satisfy the following three requirements:

i) they must have eigenvectors which exhibit maximal mixing between the neutrino and the sterile neutrino;

ii) the neutrino must have a light see-saw suppressed eigenvalue, as in the usual see-saw mechanism; and finally,

iii) the eigenvalues of the neutrino and the sterile neutrino must not be equal as we require non-degenerate masses to get oscillations.

Note that we will concentrate on the single generation case for simplicity. In order to 
solve the solar and atmospheric neutrino problems simultaneously we of course need at least two generations of maximally mixed active and sterile neutrinos with $\delta m^{2}$ values given by Eqs.11 and 2. We also need intergenerational mixing to be small in order to not perturb the active-sterile maximal mixing scenario too much. Recall that small intergenerational mixing is also suggested by the observed form of the Kobayashi-Maskawa matrix in the quark sector and by the LSND experiment. So, one can solve all three neutrino anomalies by replicating the one-generation neutrino sector we will construct below and introducing small intergenerational mixing.

In order to get see-saw suppressed eigenvalues, we must in addition to our sterile neutrino, introduce at least one exotic heavy neutral fermion into our model. In fact the simplest mass matrix which fulfils the above properties is the following $3 \times 3$ matrix in $\left\{\nu_{L},\left(\nu_{R}\right)^{c},\left(N_{R}\right)^{c}\right\}$ space, shown below together with its diagonalisation:

$$
\Lambda\left(\begin{array}{ccc}
0 & 0 & a \\
0 & 0 & a \\
a & a & 1
\end{array}\right) \rightarrow \Lambda\left(\begin{array}{ccc}
0 & 0 & 0 \\
0 & -2 a^{2} & 0 \\
0 & 0 & 1+2 a^{2}
\end{array}\right),
$$

where the diagonalising unitary matrix $U$ has the form,

$$
U=\frac{1}{\sqrt{2}}\left(\begin{array}{ccc}
1 & 1 & \sqrt{2} a \\
-1 & 1 & \sqrt{2} a \\
0 & -2 a & \sqrt{2}
\end{array}\right) .
$$

Note that we have denoted our sterile neutrino as $\nu_{R}$ and our massive exotic neutrino (which is also sterile) as $N_{R}$, and in the interests of simplicity have expressed all masses in terms of $\Lambda$, the mass of the heavy exotic neutrino; i.e. $a=m_{a} / \Lambda$. Thus $a$ is very small and we are hence able to express the eigenvalues and eigenvectors in series expanded form to order $a^{3}$ and $a^{2}$ respectively. Although not necessary we have for simplicity also assumed that our masses are real. The mass eigenstates $\left\{\nu_{1}, \nu_{2}, \nu_{3}\right\}$, corresponding to the mass matrix shown in Eq(6) are given by $\left\{\nu_{1}, \nu_{2}, \nu_{3}\right\}=\left\{\nu_{L},\left(\nu_{R}\right)^{c},\left(N_{R}\right)^{c}\right\} U$.

The above $3 \times 3$ matrix is however unsuitable for the purposes of model building as it requires that two of the off-diagonal entries appearing in the mass matrix, labelled $a$ in 
Eq(G), be equal. This is essentially a requirement that the mass terms $\bar{N}_{R} \nu_{L}$ and $\bar{N}_{R}\left(\nu_{R}\right)^{c}$ be equal, which is only possible if we have an exact unbroken left-right symmetry. Such a left-right symmetric theory would however be at odds with the standard model and hence phenomenologically ruled out. We must hence turn our attention towards suitable $4 \times 4$ matrices.

The $2 \times 2$ null matrix appearing in the top left hand corner of the above $3 \times 3$ matrix turns out to be a fundamental requirement for any matrix which is to provide us with the required properties. It is essentially a generalisation to higher dimension of the null entry appearing in the $2 \times 2$ matrix in $\left\{\nu_{L},\left(N_{R}\right)^{c}\right\}$ space featuring in the usual see-saw mechanism,

$$
\left(\begin{array}{cc}
0 & m^{\dagger} \\
m & M
\end{array}\right) .
$$

Any plausible $4 \times 4$ mass matrix is therefore expected to be a particular case of the following general matrix form in $\left\{\nu_{L},\left(\nu_{R}\right)^{c}, N_{L},\left(N_{R}\right)^{c}\right\}$ space,

$$
\left(\begin{array}{cccc}
0 & 0 & m_{a} & m_{b} \\
0 & 0 & m_{c} & m_{d} \\
m_{a} & m_{c} & \Lambda_{1} & \Lambda \\
m_{b} & m_{d} & \Lambda & \Lambda_{2}
\end{array}\right) .
$$

In this case we have introduced two heavy sterile exotic neutral fermions $N_{L}$ and $N_{R}$ with both Dirac $\Lambda$, and Majorana $\Lambda_{1}$ and $\Lambda_{2}$, mass terms. For simplicity we will again express each of the masses in terms of the heavy Dirac mass $\Lambda$ by writing $a, b, c, d=m_{a, b, c, d} / \Lambda$, $\lambda_{1}=\Lambda_{1} / \Lambda$, and $\lambda_{2}=\Lambda_{2} / \Lambda$. This notation is useful because as we will soon see our Dirac mass term $\Lambda$ is constrained to be much heavier than all of the other mass terms thus allowing us to series expand in terms of $a, b, c, d, \lambda_{1}$, and $\lambda_{2}$. Our task of finding a suitable maximally mixed neutrino model is thus essentially a task of finding suitable constraints on, or symmetries between, the masses $m_{a}, m_{b}, m_{c}$ and $m_{d}$.

Four possibilities were considered. The first is the simple case where all four masses are equal $m_{a}=m_{b}=m_{c}=m_{d}$. However like the $3 \times 3$ model previously considered this requires 
that there be an unbroken left-right symmetry which is undesirable from a model building point of view. Another possibility is to impose a symmetry whereby $m_{a}=m_{d}$ and $m_{b}=m_{c}$; a possibility which has already been investigated in the context of mirror models 16 .

The two mass matrices which we will be interested in require no symmetry between the mass terms. Instead they require the constraint that either $m_{a}=m_{d}=0$ or $m_{b}=m_{c}=0$, with the other two parameters being free subject to the condition that they be much less than $\Lambda$. We thus we need only explain the absence of two of the four masses, $m_{a}, m_{b}, m_{c}$ and $m_{d}$, appearing in $\mathrm{Eq}(\mathrm{9})$, hence these matrices are desirable from a model building perspective. Expanding in terms of the small parameters $a, b, c, d, \lambda_{1}$, and $\lambda_{2}$, these two matrices give the following diagonalisations,

$$
\begin{aligned}
& \left(\begin{array}{cccc}
0 & 0 & 0 & b \\
0 & 0 & c & 0 \\
0 & c & \lambda_{1} & 1 \\
b & 0 & 1 & \lambda_{2}
\end{array}\right) \rightarrow \\
& \left(\begin{array}{cccc}
b c+\frac{1}{2}\left(b^{2} \lambda_{1}+c^{2} \lambda_{2}\right) & 0 & 0 & 0 \\
0 & -b c+\frac{1}{2}\left(b^{2} \lambda_{1}+c^{2} \lambda_{2}\right) & 0 & 0 \\
0 & 0 & 1+\frac{1}{2}\left(\lambda_{1}+\lambda_{2}\right) & 0 \\
0 & 0 & 0 & -1+\frac{1}{2}\left(\lambda_{1}+\lambda_{2}\right)
\end{array}\right)
\end{aligned}
$$

where in this case the diagonalising unitary matrix $U$ takes the form,

$$
U=\frac{1}{\sqrt{2}}\left(\begin{array}{cccc}
-1-\frac{1}{4}\left(\frac{b}{c} \lambda_{1}-\frac{c}{b} \lambda_{2}\right) & -1+\frac{1}{4}\left(\frac{b}{c} \lambda_{1}-\frac{c}{b} \lambda_{2}\right) & b & -b \\
1-\frac{1}{4}\left(\frac{b}{c} \lambda_{1}-\frac{c}{b} \lambda_{2}\right) & -1-\frac{1}{4}\left(\frac{b}{c} \lambda_{1}-\frac{c}{b} \lambda_{2}\right) & c & c \\
b & b & 1+\frac{1}{4}\left(\lambda_{1}-\lambda_{2}\right) & -1+\frac{1}{4}\left(\lambda_{1}-\lambda_{2}\right) \\
-c & c & 1-\frac{1}{4}\left(\lambda_{1}-\lambda_{2}\right) & 1+\frac{1}{4}\left(\lambda_{1}-\lambda_{2}\right)
\end{array}\right)
$$

and, 


$$
\begin{gathered}
\left(\begin{array}{cccc}
0 & 0 & a & 0 \\
0 & 0 & 0 & d \\
a & 0 & \lambda_{1} & 1 \\
0 & d & 1 & \lambda_{2}
\end{array}\right) \rightarrow \\
\left(\begin{array}{cccc}
a d+\frac{1}{2}\left(a^{2} \lambda_{1}+d^{2} \lambda_{2}\right) & & \\
0 & 0 & 0 & 0 \\
0 & -a d+\frac{1}{2}\left(a^{2} \lambda_{1}+d^{2} \lambda_{2}\right) & 0 & 0 \\
0 & 0 & 1+\frac{1}{2}\left(\lambda_{1}+\lambda_{2}\right) & 0 \\
0 & 0 & 0 & -1+\frac{1}{2}\left(\lambda_{1}+\lambda_{2}\right)
\end{array}\right)
\end{gathered}
$$

where our diagonalising unitary matrix $U$ looks like,

$$
U=\frac{1}{\sqrt{2}}\left(\begin{array}{cccc}
-1+\frac{1}{4}\left(\frac{d}{a} \lambda_{1}-\frac{a}{d} \lambda_{2}\right) & 1+\frac{1}{4}\left(\frac{d}{a} \lambda_{1}-\frac{a}{d} \lambda_{2}\right) & a & -a \\
1+\frac{1}{4}\left(\frac{d}{a} \lambda_{1}-\frac{a}{d} \lambda_{2}\right) & 1-\frac{1}{4}\left(\frac{d}{a} \lambda_{1}-\frac{a}{d} \lambda_{2}\right) & d & d \\
-a & -a & 1+\frac{1}{4}\left(\lambda_{1}-\lambda_{2}\right) & 1-\frac{1}{4}\left(\lambda_{1}-\lambda_{2}\right) \\
d & -d & 1-\frac{1}{4}\left(\lambda_{1}-\lambda_{2}\right) & -1-\frac{1}{4}\left(\lambda_{1}-\lambda_{2}\right)
\end{array}\right) .
$$

Note that in the above expressions we have expanded all but the first two entries in the diagonalised matrices to second order in the small parameters; the first two enteries have been expanded to higher order, owing to their increased phenomenological interest. The maximally mixed mass eigenstates $\left\{\nu_{1}, \nu_{2}, \nu_{3}, \nu_{4}\right\}$ arising from the above matrices are in these cases given by,

$\left\{\nu_{1}, \nu_{2}, \nu_{3}, \nu_{4}\right\}=\left\{\nu_{L},\left(\nu_{R}\right)^{c}, N_{L},\left(N_{R}\right)^{c}\right\} U$

From the above diagonalisation it can be seen that we will obtain our desired eigenvectors and eigenvalues if we have the mass hierarchy $\lambda_{1}, \lambda_{2}, a, b, c, d<<1$, and the condition that the ratios $\lambda_{1} b / c, \lambda_{1} d / a, \lambda_{2} c / b$, and $\lambda_{2} a / d$ be much less than unity. Note that the $\Lambda_{1}$ and $\Lambda_{2}$ Majorana mass terms are required to break the degeneracy which would otherwise exist between the light eigenvalues.

In the next section we will show one way in which the standard model can be extended to account for and explain the mass matrices shown in $\mathrm{Eq}(10)$ and (12). These two matrices 
are very much analogous, thus in the interests of simplicity we will initially concentrate on developing a model which gives us a mass matrix like that shown in Eq(10). Models which will produce the mass matrix shown in $\mathrm{Eq}(12)$ will follow trivially.

\section{The Model}

To build an appropriate model we must first of all be able to explain the absence of the usual Dirac and Majorana mass terms one normally expects to be associated with the neutrino $\nu_{L}$ and its sterile partner $\nu_{R}$, i.e. we must explain the $2 \times 2$ null matrix. The nonexistence of these mass terms can be explained if the Higgs scalars required to generate these masses are absent.

In addition to explaining the absence of these conventional mass terms, we must also explain the absence of two of the four possible couplings between $N_{R, L}$ and $\nu_{R, L}$. Both of these problems can be solved by introducing a new $U(1)$ gauge group with a quantum number which we will label $T$. This new $U(1)_{T}$ symmetry will not only allow us to explain the absence of $\nu_{L}-\nu_{R}$ mass couplings, but will also allow us to differentiate between the mass terms $\left(m_{a} \bar{l}_{L}\left(N_{L}\right)^{c}, m_{d} \bar{N}_{R}\left(\nu_{R}\right)^{c}\right)$ and $\left(m_{b} \bar{l}_{L} N_{R}, m_{c} \bar{N}_{L} \nu_{R}\right)$, as each class of mass term will transform differently under a $U(1)_{T}$ gauge transformation.

The desired neutral mass matrix can be obtained if we assign the following $S U(3)_{c} \otimes$ $S U(2)_{L} \otimes U(1)_{Y} \otimes U(1)_{T}$ group representations to our neutral fermions,

$$
\begin{aligned}
l_{L} & \sim(1,2,-1,0) \\
\nu_{R} & \sim(1,1,0,2) \\
N_{L} & \sim(1,1,0,1) \\
N_{R} & \sim(1,1,0,1) .
\end{aligned}
$$

The complete list of possible Dirac and Majorana type mass terms which can be generated between these neutral fermions are given below:

$$
\overline{l_{L}}\left(N_{L}\right)^{c} \sim(1,2,1,-1)
$$




$$
\begin{aligned}
\bar{N}_{L} \nu_{R} & \sim(1,1,0,1) \\
\overline{l_{L}} N_{R} & \sim(1,2,1,1) \\
\bar{N}_{R}\left(\nu_{R}\right)^{c} & \sim(1,1,0,-3) \\
\bar{N}_{L} N_{R} & \sim(1,1,0,0) \\
\bar{N}_{L}\left(N_{L}\right)^{c} & \sim(1,1,0,-2) \\
\bar{N}_{R}\left(N_{R}\right)^{c} & \sim(1,1,0,-2) \\
\overline{l_{L}}\left(l_{L}\right)^{c} & \sim(1,3,2,0) \\
\overline{\nu_{R}}\left(\nu_{R}\right)^{c} & \sim(1,1,0,-4) \\
\overline{l_{L}} \nu_{R} & \sim(1,2,1,2) .
\end{aligned}
$$

In our mass matrix we require that the following and only the following mass terms exist: $m_{b} \bar{N}_{R} l_{L}, m_{c} \bar{N}_{L} \nu_{R}, \Lambda \bar{N}_{L} N_{R}, \Lambda_{1} \bar{N}_{L}\left(N_{L}\right)^{c}$, and $\Lambda_{2} \bar{N}_{R}\left(N_{R}\right)^{c}$.

We can generate these terms to the exclusion of the other terms listed in Eq(16) by proposing the existence of the Higgs system,

$\phi_{1} \sim(1,1,0,1)$

$\phi_{2} \sim(1,2,1,1)$

$\phi_{3} \sim(1,1,0,2)$,

with vacuum expectation values,

$$
\begin{aligned}
& \left\langle\phi_{1}\right\rangle=v_{1} \\
& \left\langle\phi_{2}\right\rangle=\left(\begin{array}{l}
0 \\
v_{2}
\end{array}\right) \\
& \left\langle\phi_{3}\right\rangle=v_{3} .
\end{aligned}
$$

We have made the above assignment in such a way that the Dirac mass $\Lambda$ associated with the mass term $\bar{N}_{L} N_{R}$ is a bare mass. This allows for a natural explanation for our required 
mass hierarchy, $\Lambda>m_{b}, m_{c}, \Lambda_{1}, \Lambda_{2}$, if the ratios $v_{1} / \Lambda, v_{2} / \Lambda$, and $v_{3} / \Lambda$ are much less than unity.

Having demonstrated how one might go about generating the required masses for the neutral fermion sector we must now turn our attention to the charged fermion sector. We wish to generate our charged fermion masses through Yukawa couplings involving the $\phi_{2} \sim$ $(1,2,1,1)$ Higgs as opposed to the usual standard model Higgs $\phi \sim(1,2,1,0)$. Our charged fermions are thus given the following group assignments,

$$
\begin{aligned}
l_{L} & \sim(1,2,-1,0) \\
e_{R} & \sim(1,1,-2,-1) \\
q_{L} & \sim(3,2,1 / 3,0) \\
u_{R} & \sim(3,1,4 / 3,1) \\
d_{R} & \sim(3,1,-2 / 3,-1),
\end{aligned}
$$

and our fermion mass generating Yukawa interactions take the form,

$$
\begin{aligned}
\mathcal{L}_{y u k}=g_{1} \bar{N}_{L} \phi_{1}^{*} \nu_{R} & +g_{2} \overline{l_{L}} \phi_{2}^{c} N_{R}+g_{3} \bar{N}_{L} \phi_{3}\left(N_{L}\right)^{c}+g_{4} \bar{N}_{R} \phi_{3}\left(N_{R}\right)^{c} \\
& +g_{5} \overline{l_{L}} \phi_{2} e_{R}+g_{6} \overline{q_{L}} \phi_{2}^{c} u_{R}+g_{7} \overline{q_{L}} \phi_{2} d_{R}+\text { h.c. }
\end{aligned}
$$

If the vacuum expectation values of $\phi_{1}, \phi_{2}$, and $\phi_{3}$ are all of similar order, then the smallness of the neutrino mass can be explained in a manner analogous to that given in the standard see-saw mechanism, we retain the usual see-saw relation $m_{\nu_{L, R}} m_{N_{L, R}} \simeq m_{e, u, d}^{2}$.

From $\mathrm{Eq}(17)$ it can be seen that the vacuum expectation values of $\phi_{1}$ and $\phi_{2}$ will spontaneously break our $U(1)_{T}$ symmetry. It is thus of interest whether our $U(1)_{T}$ group can be a local symmetry, or whether it is confined to be a global symmetry only. If it is a local symmetry then the Majoron-like Goldstone boson 31 will be eaten by the putative $U(1)_{T}$ gauge boson. If however it is a global symmetry we have to ensure that the Goldstone boson does not render our theory phenomenologically unacceptable. For our gauge group to be locally symmetric it must satisfy each of the five $U(1)_{T}$ anomaly cancellation conditions 32 : $[S U(3)]^{2} U(1)_{T},\left[S U(2)_{L}\right]^{2} U(1)_{T},\left[U(1)_{Y}\right]^{2} U(1)_{T}, U(1)_{Y}\left[U(1)_{T}\right]^{2}$, and $\left[U(1)_{T}\right]^{3}$. It turns out 
that there is no way of assigning our new quantum number $T$ to our fermion sector in such a way as to satisfy each of these anomaly cancellation conditions whilst still giving the required neutral fermion mass matrix. Our $U(1)_{T}$ symmetry is hence constrained to be a global symmetry only, and we must analyse the imaginary components of the Higgs sector to check for any phenomenological difficulties which may be associated with the Goldstone boson.

Such phenomenological difficulties would arise if it was found that our Goldstone boson participated in interactions involving quarks or charged leptons, or if it coupled to the $Z$ boson. This would happen if the Goldstone boson field contained an admixture of $\phi_{2}$ in addition to $\phi_{1}$ and $\phi_{3}$. Note from $\operatorname{Eq}(20)$ that $\phi_{1}$ and $\phi_{3}$ do not couple to charged fermions, and that $\phi_{1}$ and $\phi_{3}$ have trivial electroweak quantum numbers and hence do not couple to the $Z$ boson. Since $\phi_{1}$ and $\phi_{3}$ have trivial electroweak quantum numbers, it is fairly clear that the unphysical Goldstone boson eaten by the $Z$ must be purely from $\phi_{2}$ and thus the physical Goldstone boson associated with $U(1)_{T}$ breakdown will be constructed purely from $\phi_{1}$ and $\phi_{3}$. We now explicitly check this reasoning by calculating the neutral mass matrices associated with the shifted Higgs fields.

We begin by writing the shifted Higgs fields as,

$$
\begin{aligned}
& \phi_{1}=v_{1}+\sigma_{1}+i \sigma_{1}^{\prime} \\
& \phi_{2}=\left(\begin{array}{c}
0 \\
v_{2}+\sigma_{2}+i \sigma_{2}^{\prime}
\end{array}\right) \\
& \phi_{3}=v_{3}+\sigma_{3}+i \sigma_{3}^{\prime},
\end{aligned}
$$

where the charged Higgs field $\phi_{2}^{+}$has been set to zero since we know it is eaten by the $W^{+}$ boson. Upon substituting $\mathrm{Eq}(21)$ into the following expression for the potential of our three component Higgs sector,

$$
\begin{aligned}
V=\sum_{i=1,2,3} \lambda_{i}\left(\phi_{i}^{\dagger} \phi_{i}\right)^{2} & +\lambda_{4}\left(\phi_{1}^{\dagger} \phi_{1}\right)\left(\phi_{2}^{\dagger} \phi_{2}\right)+\lambda_{5}\left(\phi_{1}^{\dagger} \phi_{1}\right)\left(\phi_{3}^{\dagger} \phi_{3}\right)+\lambda_{6}\left(\phi_{2}^{\dagger} \phi_{2}\right)\left(\phi_{3}^{\dagger} \phi_{3}\right) \\
& -\sum_{i=1,2,3} n_{i}^{2}\left(\phi_{i}^{\dagger} \phi_{i}\right)+a \phi_{3}^{*} \phi_{1} \phi_{1}+a \phi_{3} \phi_{1}^{*} \phi_{1}^{*},
\end{aligned}
$$


and simplifying using the following constraints between $v_{1}, v_{2}$ and $v_{3}$, (the minimisation conditions of $\mathrm{Eq}(22))$,

$$
\begin{aligned}
& \frac{\delta V}{\delta \phi_{1}}=0=4 \lambda_{1} v_{1}^{3}+2 \lambda_{4} v_{1} v_{2}^{2}+2 \lambda_{5} v_{1} v_{3}^{2}-2 n_{1}^{2} v_{1}+4 a v_{3} v_{1} \\
& \frac{\delta V}{\delta \phi_{2}}=0=4 \lambda_{2} v_{2}^{3}+2 \lambda_{4} v_{1}^{2} v_{2}+2 \lambda_{6} v_{2} v_{3}^{2}-2 n_{2}^{2} v_{2} \\
& \frac{\delta V}{\delta \phi_{3}}=0=4 \lambda_{3} v_{3}^{3}+2 \lambda_{5} v_{1}^{2} v_{3}+2 \lambda_{6} v_{2}^{2} v_{3}-2 n_{3}^{2} v_{3}+2 a v_{1}^{2}
\end{aligned}
$$

we obtain the following neutral Higgs mass matrices in $\left(\sigma_{1}, \sigma_{2}, \sigma_{3}\right)$ and $\left(\sigma_{1}^{\prime}, \sigma_{2}^{\prime}, \sigma_{3}^{\prime}\right)$ space respectively,

$$
\begin{aligned}
& \left(\begin{array}{ccc}
4 \lambda_{1} v_{1}^{2} & 2 \lambda_{4} v_{1} v_{2} & 2 \lambda_{5} v_{1} v_{3}+2 a v_{1} \\
2 \lambda_{4} v_{1} v_{2} & 4 \lambda_{2} v_{2}^{2} & 2 \lambda_{6} v_{2} v_{3} \\
2 \lambda_{5} v_{1} v_{3}+2 a v_{1} & 2 \lambda_{6} v_{2} v_{3} & 4 \lambda_{3} v_{3}^{2}+a v_{1}^{2} / v_{3}
\end{array}\right) \\
& \left(\begin{array}{ccc}
-4 a v_{3} & 0 & 2 a v_{1} \\
0 & 0 & 0 \\
2 a v_{1} & 0 & -a v_{1}^{2} / v_{3}
\end{array}\right)
\end{aligned}
$$

Upon diagonalising $\mathrm{Eq}(27)$ we find that we are left with two zero eigenvalues, one being associated purely with the $\sigma_{2}^{\prime}$ boson, and the other being associated with one of the two mixed $\sigma_{1}^{\prime}-\sigma_{3}^{\prime}$ eigenstates. The remaining orthogonal $\sigma_{1}^{\prime}-\sigma_{3}^{\prime}$ mixed state acquires a mass. The $\sigma_{2}^{\prime}$ Goldstone boson can thus be gauged away by an $S U(2)_{L} \times U(1)_{Y}$ local gauge transformation and hence there will be none of the phenomenological difficulties otherwise associated with a physical Goldstone boson containing an admixture of $\sigma_{2}^{\prime}$. Instead the physical Goldstone boson which inevitably arises from the breakdown of the global symmetry $U(1)_{T}$ is a mixed $\sigma_{1}^{\prime}-\sigma_{3}^{\prime}$ eigenstate involved exclusively in fermion interactions involving at least one of our exotic heavy neutral fermions $N_{L}$ and $N_{R}$. It thus poses no phenomenological problems. Likewise the massive CP-odd boson, the orthogonal partner to our physical Goldstone boson, also presents no difficulties as it is both heavy and like the physical Goldstone 
boson exclusively associated with interactions involving at least one of our heavy neutral fermions. Note that possible cosmological consequences of the Goldstone boson have yet to be explored.

Finally we briefly consider how the mass matrix in $\mathrm{Eq}(12)$ can similarly be explained using a $U(1)_{T}$ extension to the standard model. To obtain this alternative maximal mixing inducing mass matrix we need to include the mass terms $m_{a} \overline{l_{L}}\left(N_{L}\right)^{c}$, and $m_{d} \bar{N}_{R}\left(\nu_{R}\right)^{c}$, but exclude the $m_{c} \bar{N}_{L} \nu_{R}$, and $m_{b} \bar{l}_{L} N_{R}$ interactions. This is most easily done by changing the $U(1)_{T}$ group assignments of our exotic neutral fermions $N_{L}$ and $N_{R}$ from $T=1$ to $T=-1$. This is only a minor alteration to the above model which does not effect the Higgs sector or the subsequent analysis in any significant way.

\section{Conclusion}

We have effectively found two independent $4 \times 4$ mass matrices which upon diagonalisation give rise to approximately maximal $\nu_{L}-\nu_{R}$ mixing 33 . Furthermore, a see-saw-like mechanism is used in order to explain why two of the four neutrino mass eigenvalues per generation are tiny. By employing such mass matrices for the first two generations, the solar and atmospheric neutrino anomalies can be solved by maximal active-sterile neutrino oscillations (and the LSND result can be incorporated by introducing small intergenerational mixing). (As explained in the Introduction, the potential problem thus created for Big Bang Nucleosynthesis is elegantly solved by using active-sterile neutrino oscillations in the early universe to create large neutrino asymmetries 28 .)

The two models developed to theoretically justify these two mass matrices both involve the introduction of an extended Higgs and neutral fermion sector, and a new global gauge group $U(1)_{T}$. The spontaneous breakdown of $U(1)_{T}$ gives rise to a singlet-Majoron-like Goldstone boson that poses no phenomenological difficulties.

The models presented in this paper should be contrasted with the Exact Parity Model 1 . The latter solves the solar and atmospheric neutrino problems through maximally mixed 
ordinary and mirror neutrinos. While the models constructed above have fewer degrees of freedom than the Exact Parity Model, one has to conclude that the Exact Parity Model is more elegant and compelling due to the theoretical appeal of the unbroken parity symmetry. It is interesting to note that in principle cosmology could distinguish between the two scenarios. The neutrino asymmetry creation required to make both the sterile neutrino and mirror neutrino scenarios consistent with Big Bang Nucleosynthesis occurs only for certain regions of parameter space. The parameter space region for mirror neutrinos is larger than that for truly sterile neutrinos, as a comparison of the results of Ref.28 and Ref. 64 shows. It could turn out that terrestrial experiments eventually pinpoint a region that makes mirror neutrinos cosmologically consistent but truly sterile neutrinos inconsistent.

\section{Acknowledgements}

We thank R. Foot for some useful discussions. RRV is supported by the Australian Research Council. JPB is supported by the Commonwealth of Australia. 


\section{REFERENCES}

1. Homestake Collaboration, B.T. Cleveland et al., Nucl. Phys. B (Proc. Suppl.) 38, 47 (1995); Kamiokande Collab., Nucl. Phys. B (Proc. Suppl.) 38, 55 (1995); Gallex Collab., P. Anselmann et al. Phys. Lett. B327, 377 (1994); B342, 440 (1995) and B357, 237 (1995); W. Hampel et al. Phys. Lett. B388, 384 (1996); Sage Collab., J.N. Abdurashitov et al., Phys. Lett. B328, 234 (1994); and Phys. Rev. Lett. 77, 4708 (1996).

2. R. Svoboda, talk at News about SNUS, Santa Barbara ITP, December 1997, available at http://www.itp.ucsb.edu/online/snu.

3. K.S. Hirata et al., Phys. Lett. B205, 416 (1988); and B280, 146 (1992); Y. Fukada et al., Phys. Lett. B335, 237 (1994); D. Casper et al., Phys. Rev. Lett. 66, 2561 (1991); R. Becky-Szandy et al., Phys. Rev. D46, 3720 (1992); C. Berger et al., Phys. Lett. B245, 305 (1990); M. Aglietta et al. Europhys. Lett. 15, 559 (1991); W.W.M. Allison et al. Phys. Lett. B391, 491 (1997).

4. SuperKamiokande collaboration, Y. Fukuda et al., hep-ex/9803006; E. Kearns, talk at News about SNUS, Santa Barbara ITP, December 1997, available at http://www.itp.ucsb.edu/online/snu.

5. LSND Collaboration, C. Athanassopoulos et. al., Phys. Rev. Lett. 75, 2650 (1995); 77, 3082 (1996); Phys. Rev. C54, 2685 (1996); nucl-ex/9706006 (1997); nucl-ex/9709006 (1997).

6. J. Kleinfeller, Proceedings of the 17th International Conference on Neutrino Physics and Astrophysics (World Scientific, Singapore, 1997), eds. K. Enqvist, K. Huitu \& J. Maalampi, pp.193-202.

7. C. Y. Cardall \& G. Fuller, Phys. Rev. D53, 4421 (1996).

8. A. Acker \& S. Pakvasa, Phys. Lett. B397, 209 (1997).

9. CHOOZ Collaboration, M. Apollonio et al., hep-ex/9711002 (1997). 
10. G. Gratta, Proceedings of the 17th International Conference on Neutrino Physics and Astrophysics (World Scientific, Singapore, 1997), eds. K. Enqvist, K. Huitu \& J. Maalampi, pp.248-258.

11. R. Foot, R. R. Volkas and O. Yasuda, hep-ph/9802287.

12. P.F. Harrison, D.H. Perkins \& W.G. Scott, Phys. Lett. B349, 137 (1995); B374, 111 (1996); B396, 186 (1997).

13. J. T. Peltoniemi \& J. W. F. Valle, Nucl. Phys. B406, 409 (1993); D. O. Caldwell and R. N. Mohapatra, Phys. Rev. D50, 3477 (1994).

14. L. Wolfenstein, Phys. Rev. D17, 2369 (1978); Phys. Rev. D20, 2634 (1979); S. P. Mikheyev \& A. Yu. Smirnov, Nuovo Cim. C9, 17 (1986). See also, V. Barger et al., Phys. Rev. D22, 2718 (1980).

15. K. S. Babu, J. C. Pati \& F. Wilczek, Phys. Lett. B359, 351 (1995).

16. R. Foot, H. Lew \& R.R. Volkas, Phys. Lett. B272, 67 (1991) and Mod. Phys. Lett. A7, 2567 (1992); R. Foot \& R.R. Volkas, Phys. Rev. D52, 6595 (1995); R. Foot, Mod. Phys. Lett. A9,169 (1994).

17. See for example, S. M. Bilenky \& B. Pontecorvo, Phys. Rep. 41, 225 (1978); V. Barger, R. J. N. Phillips \& K. Whisnant, Phys. Rev. D24, 538 (1981); S. L. Glashow \& L. M. Krauss, Phys. Lett. B190, 199 (1987); E. Calabresu et. al., Astropart. Phys. 4, 159 (1995).

18. R. R. Volkas and Y. Y. Y. Wong, hep-ph/9803456.

19. R. Foot, R. R. Volkas \& O. Yasuda, Phys. Rev. D57, 1345 (1998); hep-ph/9710403 (Phys. Lett. B, in press); hep-ph/9801431 (Phys. Rev. D, in press).

20. P. I. Krastev \& S. T. Petcov, Phys. Rev. D53, 1665 (1996); Phys. Rev. D54, 7057 (1996); N. Hata \& P. Langacker, Phys. Rev. D56, 6107 (1997); J. N. Bahcall, M. 
Fukugita \& P. I. Krastev, Phys. Lett. B374, 1 (1996).

21. J. N. Bahcall \& M. H. Pinsonneault, Rev. Mod. Phys. 67, 1 (1995); S. Turck-Chieze \& I. Lopes, Ap. J. 408, 347 (1993); J. N. Bahcall \& H. A. Bethe, Phys. Rev. Lett. 65, 2233 (1990); J. N. Bahcall, Phys. Lett. B338, 276 (1994); A. Dar \& G. Shaviv, Ap. J. 468, 933 (1996).

22. A. Acker, S. Pakvasa, J. Learned \& T. J. Weiler, Phys. Lett. B298, 149 (1993); A. Acker \& S. Pakvasa, see Ref.8. E. Torrente-Lujan, Phys. Lett B389, 557 (1996); G. Conforto et al, Astropart. Phys. 5, 147 (1996); hep-ph/9708301; R. Foot \& R. R. Volkas, preprint UM-P-95/94, hep-ph/9510312; P. I. Krastev \& S. T. Petcov, first paper in Ref.20; P. F. Harrison, D. H. Perkins \& W. G. Scott, third paper in Ref.12.

23. K. Lande, Proceedings of the 17th International Conference on Neutrino Physics and Astrophysics (World Scientific, Singapore, 1997), eds. K. Enqvist, K. Huitu \& J. Maalampi, pp.25-37.

24. C. Arpesella et al., BOREXINO Poposal, Vols. 1 and 2, ed. G. Bellini, R. Raghavan, et al., (University of Milano, Milano, 1992).

25. A. Dolgov, Sov. J. Nucl. Phys. 33, 700 (1981); R. Barbieri \& A. Dolgov, Phys. Lett. B237, 440 (1990) and Nucl. Phys. B349, 743 (1991); K. Enqvist, K. Kainulainen \& M. Thomson, Nucl. Phys. B373, 498 (1992); J. Cline, Phys. Rev. Lett. 68, 3137 (1992); X. Shi, D. N. Schramm \& B. D. Fields, Phys. Rev. D48, 2568 (1993).

26. K. Olive, Proceedings of the 17th International Conference on Neutrino Physics and Astrophysics (World Scientific, Singapore, 1997), eds. K. Enqvist, K. Huitu \& J. Maalampi, pp. $445-460$.

27. R. Foot, M. J. Thomson \& R.R. Volkas, Phys. Rev. D53, 5349 (1996); X. Shi, Phys. Rev. D54, 2753 (1996).

28. R. Foot \& R.R. Volkas, Phys. Rev. D55, 5147 (1997). 
29. S. M. Bilenky and S. T. Petcov, Rev. Mod. Phys. 59, 671 (1987); C. Giunti, C. W. Kim \& V. W. Lee, Phys. Rev. D46, 3034 (1992).

30. M. Gell-Mann, P. Ramond, \& R. Slansky, in Supergravity, eds. P. Van Nieuwenhuizen and D. Z. Freedman, North Holland, (1979); T. Yanagida, in Proc. Workshop on Unified Theory and Baryon Number of the Universe, eds. A. Sawada and H. Sugawara (KEK 1979); R. N. Mohapatra \& G. Senjanovic, Phys. Rev. Lett. 44, 912 (1980).

31. Y. Chikashige, R. N. Mohapatra, \& R. D. Peccei, Phys. Lett. B98, 265 (1981).

32. C. Bouchiat, J. Iliopoulos, \& P. Meyer, Phys. Lett. B38, 519 (1972); D. Gross, \& R. Jackiw, Phys. Rev. D6, 447 (1972); H. Georgi, \& S. L. Glashow, Phys. Rev. D6, 429 (1972).

33. The superstring motivated scenario considered in R. N. Mohapatra \& J. W. F. Valle, Phys. Lett. B177, 47 (1986) also features maximal mixing between active and sterile neutrinos in the context of the "just-so" solution to the solar neutrino problem. While the mass matrix considered by Mohapatra and Valle bears some similarity to those we have constructed, the two scenarios are theoretically distinct because the neutrino sectors are embedded in completely different theories. For example, supersymmetry breaking appears to be required by Mohapatra and Valle in order to produce Majorana rather than Dirac eigenstates, a phenomenon we obviously do not use. We thank one of the referees for bringing this reference to our attention.

34. R. Foot \& R. R. Volkas, Astropart. Phys. 7, 283 (1997). 